\title{
Lie Group Method for Studying the Heat Generation Effect on Free- convection Laminar Boundary-layer Flow Over a Vertical Flat Plate Abd-el-Malek MB ${ }^{1,3 *}$, Badran NA ${ }^{1}$, Hassan $\mathrm{HS}^{2}$ and Abbas $\mathrm{HH}^{2}$
}

${ }^{1}$ Department of Engineering Mathematics and Physics, Faculty of Engineering, Alexandria University, Alexandria 21544, Egypt

${ }^{2}$ Department of Basic and Applied Science, Arab Academy for Science, Technology and Maritime Transport, P.O. BOX 1029 Alexandria, Egypt

${ }^{3}$ Department of Mathematics and Actuarial Science, The American University in Cairo, New Cairo 11835, Egypt

\begin{abstract}
The nonlinear equations of heat and mass transfer in two-dimensional free-convection, laminar, boundary layer flow of a viscous incompressible fluid over a vertical plate with thermophoresis and heat generation effect have been considered. We apply Lie-group method for determining symmetry reductions of partial differential equations. Liegroup method starts out with a general infinitesimal group of transformations under which the given partial differential equations are invariant. The determining equations are a set of linear differential equations, the solution of which gives the transformation function or the infinitesimals of the dependent and independent variables. After the group has been determined, a solution to the given partial differential equations may be found from the invariant surface condition such that its solution leads to similarity variables that reduce the number of independent variables of the system. The effect of the heat generation parameter He, the Prandtl number Pr, the Schimted number Sc, the thermophoretic parameter $\tau$, the solutal Grashof number $\mathrm{Gc}$ and the thermal Grashof number $\mathrm{Gr}$ on velocity, concentration and temperature have been studied and the results are plotted.
\end{abstract}

Keywords: Lie-group; Similarity solutions; Thermophoresis; Heat generation; Free convection

\section{Nomenclature}

$a, b$ : Constants

$\bar{C}:$ Concentration of the substance in the fluid inside the boundary layer

$\mathrm{C}_{\infty}$ : Concentration of the fluid in the free stream air

$\mathrm{C}_{w}$ : Concentration of the fluid beside the plate

$c_{\rho}:$ Specific heat of the fluid

$D$ : Diffusion coefficient

Gc : Solutal Grashof number

Gr : Thermal Grashof number

$g$ : Acceleration due to gravity

$\mathrm{He}$ : Heat generation parameter

$k$ : Thermal conductivity of fluid

Pr : Prandtl number

$Q$ : Heat generation constant

Sc : Schimted number

$\bar{T}$ : Temperature of the fluid inside the boundary layer

$T_{\infty}$ : Temperature of the fluid in the free stream air

$T_{r}$ : Some reference temperature

$T_{w}$ : Temperature of the fluid in the plate

$U_{1}$ : Characteristic velocity

$\bar{u}$ : Velocity component along $\bar{x}$ direction

$V_{\bar{T}}:$ Thermophoretic velocity

$\bar{v}$ : Velocity component along $\bar{y}$ direction

$\bar{x}, \bar{y}:$ Space coordinates

\section{Greek symbols}
$\beta^{*}$ : Coefficient of expansion with concentration
$\beta$ : Coefficient of thermal expansion
$\rho:$ Density of the fluid
$\lambda:$ Small parameter
$v$ : Kinematic viscosity of the fluid
$k:$ Thermophoretic coefficient
$k v$ : Thermophoretic diffusivity
$\tau:$ Thermophoretic parameter

\section{Introduction}

Thermophoresis is a phenomenon which causes small particles to be driven away from a hot surface and towards a cold one. Small particles, such as dust, when suspended in a gas with a temperature gradient, experience a force in the direction opposite to the temperature gradient [1].

The velocity acquired by the particles is called the thermophoretic velocity and the force experienced by the suspended particles due to the temperature gradient is known as the thermophoretic force. The magnitudes of the thermophoretic force and velocity are proportional

*Corresponding author: Abd-el-Malek MB, Department of Engineering Mathematics and Physics, Faculty of Engineering, Alexandria University, Alexandria 21544, Egypt, Tel: 01000066496; E-mail: minab@aucegypt.edu

Received August 10, 2015; Accepted October 06, 2015; Published October 12, 2015

Citation: Abd-el-Malek MB, Badran NA, Hassan HS, Abbas HH (2015) Lie Group Method for Studying the Heat Generation Effect on Free-convection Laminar Boundary-layer Flow Over a Vertical Flat Plate. J Generalized Lie Theory Appl 9 : 232. doi:10.4172/1736-4337.1000232

Copyright: () 2015 Abd-el-Malek MB, et al. This is an open-access article distributed under the terms of the Creative Commons Attribution License, which permits unrestricted use, distribution, and reproduction in any medium, provided the original author and source are credited. 
to the temperature gradient, thermal conductivity of aerosol particles and the carrier gas, thermophoretic coefficient and the heat capacity of the gas. This phenomenon has many practical applications in removing small particles from gas streams, in determining exhaust gas particle trajectories from combustion devices, prevention of fouling and corrosion in heat exchangers and turbines, semiconductor manufacture and ceramic powder production.

Brock [2] explained the disagreement between experimental results and aerosol thermal force theories for regimes from continuum toward free-molecule through the application of first-order slip-flow boundary conditions. In his work, the influence of the convective flow on the thermal force is explored through a perturbation technique. $\mathrm{He}$ developed an expression for the thermophoretic force for continuum regime.

Brock [3] reviewed the theoretical and experimental results for the thermal force in the free molecule region. He developed a theory for the thermal force for the transition region. Experimental results appear to support his developed theory.

A laser-Doppler velocimeter (LDV) has been used by Talbot et al. [4] to study of velocity profiles in the laminar boundary layer adjacent to a heated flat plate revealed that the seed particles used for the LDV measurements were driven away from the plate surface by thermophoretic forces, causing a particle-free region within the boundary layer of approximately one half the boundary-layer thickness. Measurements of the thickness of this region were compared with particle trajectories calculated according to several theories for the thermophoretic force.

Goren [5] considered theoretically the thermophoresis of aerosol particles in the laminar compressible boundary layer on a flat plate is. The particles are attracted by a plate colder than the gas and the dust concentration drops monotonically as the wall is approached; in the absence of Brownian diffusion a nonzero concentration at the wall is found. The particles are repelled by a plate hotter than the gas and, in the absence of Brownian diffusion, the aerosol concentration profile exhibits a singularity at which the concentration rapidly approaches zero or infinity. The location of this singularity is taken to be the boundary of the dust-free layer found on heated objects.

Chiou [6] analyzed the sub-micron deposition from a laminar free convection boundary layer developing on a heated isothermal vertical cylinder. In his work, a finite-difference method has been used to theoretically calculate the local cumulative particle deposition with the thermophoretic velocity. It was demonstrated that the thermophoresis has a pronounced effect on the particle transfer but only a slight effect on the very smaller particles and that the effect increases with particle size and distance along the cylinder. Thermophoresis in natural convection for a cold vertical surface has been studied by Epstein et al. [7].

The interaction between radiation and thermophoresis in forced convection laminar boundary-layer flow over an impermeable flat plate is investigated by Jia et al. [8]. The fluid is a radiatively nonparticipating constant-property gas containing emitting, absorbing, and isotropically scattering gray aerosol particles.

Sub-micron deposition from a laminar forced convection boundary layer developing on a heated isothermal vertical cylinder has been investigated by Chiou and Cleaver [9]. Pseudo-similarity solutions have been used to calculate the local cumulative particle deposition theoretically; with the thermophoretic velocity.
Selim et al. [10] investigated the effect of surface mass flux on mixed convective flow past a heated vertical flat permeable plate with thermophoresis. A non-uniform surface mass flux through the permeable surface has been considered. The governing equations, reduced to local non-similarity boundary layer equations using suitable transformations, have been integrated employing an implicit finite difference method together with the Keller-box technique.

Alam et al. [11] studied the problem of steady, two-dimensional, laminar, hydromagnetic flow with heat and mass transfer over a semiinfinite, permeable inclined flat plate in the presence of thermophoresis and heat generation is numerically. A similarity transformation is used to reduce the governing non-linear partial differential equations into ordinary ones. The obtained locally similar equations are then solved numerically by applying Nachtsheim-Swigert shooting iteration technique with sixth-order Runge-Kutta integration scheme.

This work is concerned with the study of the effect of thermophoresis and heat generation on free-convection laminar boundary-layer flow over a vertical flat plate. Lie-group method is applied to the equations of motion, energy and diffusion for determining symmetry reductions of partial differential equations, [12-24]. Exact solutions for the temperature have been obtained and we used them to get the velocity and the concentration using MATLAP package.

\section{Mathematical Formulation of the Problem}

Consider the steady, laminar, two-dimensional free-convection boundary layer flow of a viscous incompressible fluid over a vertical plate placed in the region $\bar{y}>0$ of a Cartesian system of coordinates $O \bar{x} \bar{y}$. The plate has a uniform temperature $\mathrm{T}_{\mathrm{w}}$ and the free stream temperature is $T_{\infty}$ with $T_{w}>T_{\infty}$. The species concentration beside the plate is maintained uniform at $\mathrm{C}_{\mathrm{w}}$ and that of the ambient fluid is assumed to be $\mathrm{C}_{\infty}$. A heat source is placed within the flow to allow for possible heat generation effects. The fluid is assumed to be Newtonian and heat generating. The effects of thermophoresis are being taken into account (Figure 1).

Under the above assumptions, the governing equations of the mass, momentum, energy and concentration for such flow can be written as

Continuity Equation: $\frac{\partial \bar{u}}{\partial \bar{x}}+\frac{\partial \bar{v}}{\partial \bar{y}}=0$,

Momentum equation:

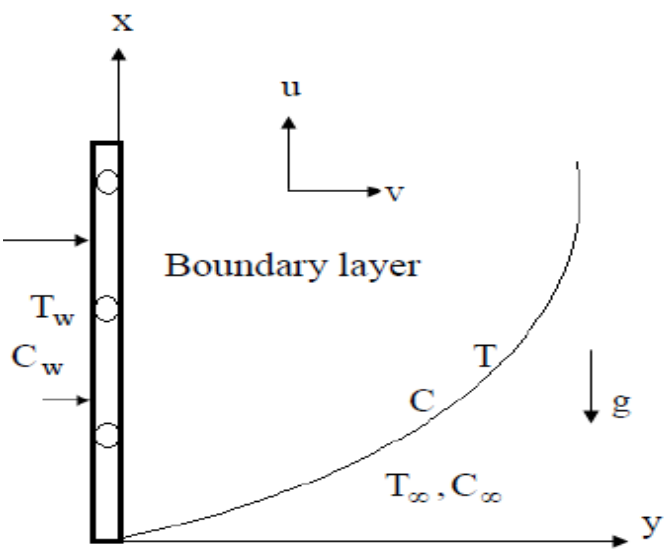

Figure 1: Physical model and coordinate system. 
$\bar{u} \frac{\partial \bar{u}}{\partial \bar{x}}+\bar{v} \frac{\partial \bar{u}}{\partial \bar{y}}=v \frac{\partial^{2} \bar{u}}{\partial \bar{y}^{2}}+g \beta\left(\bar{T}-T_{\infty}\right)+g \beta^{*}\left(\bar{C}-C_{\infty}\right)$,

Energy equation:

$\bar{u} \frac{\partial \bar{T}}{\partial \bar{x}}+\bar{v} \frac{\partial \bar{T}}{\partial \bar{y}}=\frac{k}{\rho c_{\rho}} \frac{\partial^{2} \bar{T}}{\partial \bar{y}^{2}}+\frac{Q}{\rho c_{\rho}}\left(\bar{T}-T_{\infty}\right)$,

Diffusion equation:

$\bar{u} \frac{\partial \bar{C}}{\partial \bar{x}}+\bar{v} \frac{\partial \bar{C}}{\partial \bar{y}}=D \frac{\partial^{2} \bar{C}}{\partial \bar{y}^{2}}-\frac{\partial}{\partial \bar{y}}\left(\bar{C}-C_{\infty}\right) V_{\bar{T}}$

where,

$V_{\bar{T}}=-\frac{\kappa v}{T_{r}} \frac{\partial \bar{T}}{\partial \bar{y}}$.

The boundary conditions are

(i) $\overline{\mathrm{u}}=0, \overline{\mathrm{v}}=-\mathrm{bU}_{1}, \overline{\mathrm{T}}=\mathrm{T}_{\mathrm{w}}$ and $\quad \overline{\mathrm{C}}=\mathrm{C}_{\mathrm{w}}$ at $\overline{\mathrm{y}}=0$,

(ii) $\overline{\mathrm{u}} \rightarrow 0, \overline{\mathrm{T}} \rightarrow \mathrm{T}_{\infty}$ and $\overline{\mathrm{C}} \rightarrow \mathrm{C}_{\infty}$ as $\overline{\mathrm{y}} \rightarrow \infty$

The variables in equations (2.1) - (2.4) are dimensionless according to $x=\frac{\bar{x} U_{1}}{v}, \quad y=\frac{\bar{y} U_{1}}{v}, \quad u=\frac{\bar{u}}{U_{1}}, \quad v=\frac{\bar{v}}{U_{1}}, \quad T=\frac{\bar{T}-T_{\infty}}{T_{w}-T_{\infty}}, \quad C=\frac{\bar{C}-C_{\infty}}{C_{w}-C_{\infty}}$.

Substitution from (5) and (8) into (1) - (4) yields

$$
\begin{aligned}
& u_{x}+v_{y}=0, \\
& u u_{x}+v u_{y}=u_{y y}+\frac{g \beta}{U_{1}^{2}}\left(T_{w}-T_{\infty}\right) T+\frac{g \beta^{*}}{U_{1}^{2}}\left(C_{w}-C_{\infty}\right) C, \\
& u T_{x}+v T_{y}=\frac{k}{\rho c_{\rho} U_{1}} T_{y y}+\frac{Q}{\rho c_{\rho} U_{1}} T, \\
& u C_{x}+v C_{y}=\frac{D}{U_{1}} C_{y y}+\frac{k}{T_{r}}\left(T_{w}-T_{\infty}\right)\left(C T_{y y}+C_{y} T_{y}\right),
\end{aligned}
$$

where subscripts denote partial derivatives with respect to the indicated variables.

The boundary conditions (2.6) and (2.7) will be

(i) $\mathrm{u}=0, \mathrm{v}=-\mathrm{b}, \mathrm{T}=1$ and $\mathrm{C}=1$ at $\mathrm{y}=0$,

(ii) $\mathrm{u} \rightarrow 0, \mathrm{~T} \rightarrow 0$ and $\mathrm{C} \rightarrow 0$ as $\mathrm{y} \rightarrow \infty$.

From the Continuity Equation (2.9), there exists a stream function $\psi(x, y)$ such that

$u(x, y)=\frac{\partial \psi(x, y)}{\partial y}, v(x, y)=-\frac{\partial \psi(x, y)}{\partial x}$,

which satisfies equation (2.9) identically.

Substitution from (2.15) into (2.10) - (2.12) yields

$\psi_{y} \psi_{x y}-\psi_{x} \psi_{y y}=\psi_{y y y}+G r T+G c C$,

$\psi_{y} T_{x}-\psi_{x} T_{y}=\frac{1}{\operatorname{Pr}} T_{y y}+H e T$,

$\psi_{y} C_{x}-\psi_{x} C_{y}=\frac{1}{S c} C_{y y}-\tau\left(C T_{y y}+C_{y} T_{y}\right)$,

where,

$G r=\frac{g \beta}{U_{1}^{2}}\left(T_{w}-T_{\infty}\right), G c=\frac{g \beta^{*}}{U_{1}^{2}}\left(C_{w}-C_{\infty}\right), \operatorname{Pr}=\frac{\rho c_{\rho} U_{1}}{k}, H e=\frac{Q}{\rho c_{\rho} U_{1}}, S c=\frac{U_{1}}{D}, \tau=-\frac{k}{T_{r}}\left(T_{w}-T_{\infty}\right)$.

The boundary conditions (2.13) and (2.14) will be
(i) $\quad \psi_{\mathrm{y}}=0, \psi_{\mathrm{x}}=\mathrm{b}, \mathrm{T}=1$ and $\mathrm{C}=1$ at $\mathrm{y}=0$,
(ii) $\quad \psi_{\mathrm{y}} \rightarrow 0, \mathrm{~T} \rightarrow 0$ and $\mathrm{C} \rightarrow 0$ as $\mathrm{y} \rightarrow \infty$.

\section{Solution of the Problem}

Firstly, we derive the similarity solutions using Lie-group method under which (2.16) - (2.18) and the boundary conditions (2.19) - (2.20) are invariant, and then we use these symmetries to determine the similarity variables.

\section{Lie point symmetries}

Consider the one-parameter $(\varepsilon)$ Lie group of infinitesimal transformations in $(x, y ; \psi, T, C)$ given by

$$
\begin{aligned}
& x^{*}=x+\varepsilon \varphi(x, y ; \psi, T, C)+O\left(\varepsilon^{2}\right), \\
& y^{*}=y+\varepsilon \xi(x, y ; \psi, T, C)+O\left(\varepsilon^{2}\right), \\
& \psi^{*}=\psi+\varepsilon h(x, y ; \psi, T, C)+O\left(\varepsilon^{2}\right), \\
& T^{*}=T+\varepsilon \eta(x, y ; \psi, T, C)+O\left(\varepsilon^{2}\right), \\
& C^{*}=C+\varepsilon N(x, y ; \psi, T, C)+O\left(\varepsilon^{2}\right),
\end{aligned}
$$

where " $\mathcal{E}$ " is a small parameter. The partial differential equations (2.16) - (2.18) are said to admit a symmetry generated by the vector field

$$
\Gamma \equiv \varphi \frac{\partial}{\partial x}+\xi \frac{\partial}{\partial y}+h \frac{\partial}{\partial \psi}+\eta \frac{\partial}{\partial T}+N \frac{\partial}{\partial C},
$$

if it is left invariant by the transformation $(x, y ; \psi, T, C) \rightarrow\left(x^{*}, y^{*} ; \psi^{*}, T^{*}, C^{*}\right)$. The solutions $\psi=\psi(x, y), T=T(x, y)$ and $C=C(x, y)$, are invariant under the symmetry (3.2) if

$$
\begin{aligned}
& \Phi_{\psi}=\Gamma(\psi-\psi(x, y))=0, \text { when } \psi=\psi(x, y), \\
& \Phi_{T}=\Gamma(T-T(x, y))=0, \text { when } T=T(x, y), \\
& \text { and } \\
& \Phi_{C}=\Gamma(C-C(x, y))=0, \text { when } C=C(x, y) .
\end{aligned}
$$

Assume,

$\Delta_{1}=\psi_{y} \psi_{x y}-\psi_{x} \psi_{y y}-\psi_{y y y}-G r T-G c C$,

$\Delta_{2}=\psi_{y} T_{x}-\psi_{x} T_{y}-\frac{1}{\operatorname{Pr}} T_{y y}-H e T$,

$$
\Delta_{3}=\psi_{y} C_{x}-\psi_{x} C_{y}-\frac{1}{S c} C_{y y}+\tau\left(C T_{y y}+C_{y} T_{y}\right) .
$$

A vector $\Gamma$ given by (3.2), is said to be a Lie point symmetry vector field for (2.16)-(2.18) if

$$
\left.\Gamma^{[3]}\left(\Delta_{i}\right)\right|_{\Delta_{i}=0}=0, \quad \mathrm{i}=1,2,3
$$

where,

$$
\begin{aligned}
\Gamma^{[3]} \equiv & \varphi \frac{\partial}{\partial x}+\xi \frac{\partial}{\partial y}+h \frac{\partial}{\partial \psi}+\eta \frac{\partial}{\partial T}+N \frac{\partial}{\partial C}+h^{x} \frac{\partial}{\partial \psi_{x}}+\eta^{x} \frac{\partial}{\partial T_{x}}+N^{x} \frac{\partial}{\partial C_{x}}+ \\
& h^{y} \frac{\partial}{\partial \psi_{y}}+\eta^{y} \frac{\partial}{\partial T_{y}}+N^{y} \frac{\partial}{\partial C_{y}}+h^{x y} \frac{\partial}{\partial \psi_{x y}}+h^{y y} \frac{\partial}{\partial \psi_{y y}}+ \\
& \eta^{y y} \frac{\partial}{\partial T_{y y}}+N^{y y} \frac{\partial}{\partial C_{y y}}+h^{y y y} \frac{\partial}{\partial \psi_{y y y}}
\end{aligned}
$$

is the third prolongation of $\Gamma$.

To calculate the prolongation of the given transformation, we need to differentiate (3.1) with respect to each of the variables $x$ and $y$. To do this, we introduce the following total derivatives 


$$
\begin{aligned}
& D_{x} \equiv \partial_{x}+\psi_{x} \partial_{\psi}+T_{x} \partial_{T}+C_{x} \partial_{C}+\psi_{x x} \partial_{\psi_{x}}+T_{x x} \partial_{T_{x}}+C_{x x} \partial_{C_{x}}+\psi_{x y} \partial_{\psi_{y}}+\ldots \ldots . . \\
& D_{y} \equiv \partial_{y}+\psi_{y} \partial_{\psi}+T_{y} \partial_{T}+C_{y} \partial_{C}+\psi_{y y} \partial_{\psi_{y}}+T_{y y} \partial_{T_{y}}+C_{y y} \partial_{C_{y}}+\psi_{x y} \partial_{\psi_{x}}+\ldots \ldots . .
\end{aligned}
$$

Equation (3.9) gives the following system of linear partial differential equations

$$
\begin{aligned}
& -G r \eta-G c N-\psi_{y y} h^{x}+\psi_{x y} h^{y}+\psi_{y} h^{x y}-\psi_{x} h^{y y}-h^{y y y}=0, \\
& -H e \eta-T_{y} h^{x}+\psi_{y} \eta^{x}+T_{x} h^{y}-\psi_{x} \eta^{y}-\frac{1}{\operatorname{Pr}} \eta^{y y}=0, \\
& -C_{y} h^{x}+\psi_{y} N^{x}+C_{x} h^{y}-\psi_{x} N^{y}-\frac{1}{S c} N^{y y}+\tau\left(C \eta^{y y}+T_{y y} N+C_{y} \eta^{y}+T_{y} N^{y}\right)=0
\end{aligned}
$$

The components $h^{x}, h^{y}, \eta^{x}, \eta^{y}, N^{x}, N^{y}, h^{x y}, h^{y y}, y^{y y}, \eta^{y y}, N^{y y}$ and $h^{y y}$ can be determined from the following expressions

$$
\begin{aligned}
& h^{S}=D_{S} h-\psi_{x} D_{S} \varphi-\psi_{y} D_{S} \xi, \quad \eta^{S}=D_{S} \eta-T_{x} D_{S} \varphi-T_{y} D_{s} \xi \\
& N^{s}=D_{S} N-C_{x} D_{S} \varphi-C_{y} D_{S} \xi, \quad h^{L S}=D_{S} h^{L}-\psi_{L x} D_{S} \varphi-\psi_{L y} D_{S} \xi \\
& \eta^{L S}=D_{S} \eta^{L}-T_{L x} D_{S} \varphi-T_{L y} D_{S} \xi, \quad N^{L S}=D_{S} N^{L}-C_{L x} D_{S} \varphi-C_{L y} D_{S} \xi, \\
& h^{J L}=D_{S} h^{J L}-\psi_{J x} D_{S} \varphi-\psi_{J y} D_{S} \xi,
\end{aligned}
$$

where $S, L$ and $J$ are standing for $x, y$. By substituting from (3.15) into (3.13) will lead to a large expression, then, equating to zero the coefficients of $T_{x y}, \psi_{y} T_{x y}, T_{y} T_{x y}, C_{y} T_{x y}, \psi_{y} C_{x}, T_{x}\left(\psi_{y}\right)^{2}, C_{x} T_{y} \psi_{y}$ and $T_{x} T_{y} \psi_{y}$, gives

$$
\varphi_{y}=\varphi_{\psi}=\varphi_{T}=\varphi_{C}=\eta_{C}=\xi_{\psi}=\xi_{C}=\xi_{T}=0 .
$$

By substituting (36) into (33) and equating to zero the coefficients of $\psi_{x}, \psi_{y}, T_{x}, C_{y} T_{x}, \psi_{y y}, \psi_{y} T_{x}$ and $\left(T_{y}\right)^{2}$ we get

$$
\begin{aligned}
& \eta_{y}=\eta_{x}=h_{y}=h_{c}=\eta_{\psi}=0, \\
& h_{\psi}=\varphi_{x}, \\
& \eta_{T T}=0 .
\end{aligned}
$$

By substituting (3.16)-(3.19) into (3.12) and (3.14) will lead to a system of determining equations. By solving the system of resulting determining equations in view of the invariance of the boundary conditions (2.19)-(2.20) yields

$$
\varphi=c_{1}, \quad \xi=c_{2}, \quad h=c_{3}, \quad \eta=N=0 .
$$

So, the nonlinear equations (16)-(18) have the three-parameter Lie group of point symmetries generated by

$$
\Gamma_{1} \equiv \frac{\partial}{\partial x}, \quad \Gamma_{2} \equiv \frac{\partial}{\partial y}, \quad \Gamma_{3} \equiv \frac{\partial}{\partial \psi} .
$$

The one-parameter group generated by $\Gamma_{1}, \Gamma_{2}$ and $\Gamma_{3}$ consists of translation. There aren't any non-zero commutation relations for these symmetries, where the commutation relation is given by $\left[\Gamma_{i}, \Gamma_{j}\right]=\Gamma_{i} \Gamma_{j}-$ $\Gamma_{j} \Gamma_{i}$. The finite transformations corresponding to the symmetries $\Gamma_{1}, \Gamma_{2}$ and $\Gamma_{3}$ are respectively

$$
\left.\begin{array}{l}
\Gamma_{1}: x^{*}=x+\varepsilon_{1}, y^{*}=y, \psi^{*}=\psi, T^{*}=T, C^{*}=C, \\
\Gamma_{2}: x^{*}=x, y^{*}=y+\varepsilon_{2}, \psi^{*}=\psi, T^{*}=T, C^{*}=C, \\
\Gamma_{3}: x^{*}=x, y^{*}=y, \psi^{*}=\psi+\varepsilon_{3}, T^{*}=T, C^{*}=C,
\end{array}\right\}
$$

where $\varepsilon_{1}, \varepsilon_{2}$ and $\varepsilon_{3}$ are the group parameters.

\section{One-dimensional optimal system of subalgebras of the symmetry group}

Since the symmetry Lie algebra is three-dimensional which given by the operators (3.21), we look for solutions invariant under the linear combination of these operators. All the possible invariant solutions can be obtained by determine the optimal system of one-dimensional subalgebras of the given system of partial differential equations. This is the most systematic procedure presented by Olver [16]. Following Olver's approach, we compute first the commutators of the symmetry Lie algebra (3.17), which is obtained in section 3.1, and then find the adjoint representations. The adjoint action on Lie algebras is defined by the adjoint operator given by

$$
A d_{\exp \left(\lambda \Gamma_{i}\right)}\left\langle\Gamma_{j}\right\rangle=e^{-\lambda \Gamma_{i}} \Gamma_{j} e^{\lambda \Gamma_{i}},
$$

where, $\lambda$ is a small parameter.

This operator can be rewritten in terms of Lie brackets using Campbell-Baker-Hausdorff theorem [25] as

$$
A d_{\exp \left(\lambda \Gamma_{i}\right)}\left\langle\Gamma_{j}\right\rangle=\Gamma_{j}-\lambda\left[\Gamma_{i}, \Gamma_{j}\right]+\frac{\lambda^{2}}{2 !}\left[\Gamma_{i},\left[\Gamma_{i}, \Gamma_{j}\right]\right]-.
$$

For our problem, $\Omega=\left\langle\Gamma_{1}, \Gamma_{2}, \Gamma_{3}\right\rangle$ is the Lie algebra associated with the symmetry group. The calculations of the adjoint action are summarized in Table 1.

From Table 1, it is clear that, there are not any nontrivial adjoint actions for the Lie symmetry algebra $\Omega$. To construct the onedimensional optimal system of $\Omega$, consider a general element of $\Omega$ given by

$$
\mathrm{E}=a_{1} \Gamma_{1}+a_{2} \Gamma_{2}+a_{3} \Gamma_{3},
$$

for some constants $a_{1}, a_{2}$ and $a_{3}$ and probe whether $\mathrm{E}$ can be transformed to a new element $\mathrm{E}^{\prime}$ under the general adjoint action, where $\mathrm{E}^{\prime}$ takes a simpler form than $\mathrm{E}$, [26]. Let,

$$
\mathrm{E}^{\prime}=A d_{\exp \left(\lambda \Gamma_{i}\right)}\langle E\rangle=a_{1}^{\prime} \Gamma_{1}+a_{2}^{\prime} \Gamma_{2}+a_{3}^{\prime} \Gamma_{3} .
$$

We make appropriate choice of $a$ such that the $a^{\prime}$ 's can be made 0 or 1 . We end up with simpler forms of $E$ that will constitute the one-dimensional optimal system. By substituting $\Gamma_{i}=\Gamma_{1}$ in (3.26) and dropping the primes, we get

$\mathrm{E}^{\prime}=a_{1} \Gamma_{1}+a_{2} \Gamma_{2}+a_{3} \Gamma_{3}$.

Now, equation (3.27) allows the consideration of the cases $a_{1} \neq 0$ and $a_{1}=0$.

Case (I): $a_{1} \neq 0$

Scaling the resulting operator by $a_{1}$, equation (3.27) will be

$\mathrm{E}^{\prime}=\Gamma_{1}+a \Gamma_{2}+b \Gamma_{3}$.

We can further consider the subclasses (i) $a=0, b \neq 0$, (ii) $a \neq 0$, $b=0$ and (iii) $a=b=0$. Therefore, an optimal system of one-dimensional subalgebra for this case is given by:

$\left\{\Gamma_{1}+a \Gamma_{2}+b \Gamma_{3}, \Gamma_{1}+b \Gamma_{3}, \Gamma_{1}+a \Gamma_{2}, \Gamma_{1}\right\}$, where, $a \in R$ and $b \in R$.

Case (II): $a_{1=} 0$

Using repeatedly the adjoint operation to simplify E, an optimal

\begin{tabular}{|c|c|c|c|}
\hline Ad & & $\Gamma_{2}$ & $\Gamma_{3}$ \\
\hline$\Gamma_{1}$ & $\Gamma_{1}$ & $\Gamma_{2}$ & $\Gamma_{3}$ \\
\hline$\Gamma_{2}$ & $\Gamma_{1}$ & $\Gamma_{2}$ & $\Gamma_{3}$ \\
\hline$\Gamma_{3}$ & $\Gamma_{1}$ & $\Gamma_{2}$ & $\Gamma_{3}$ \\
\hline
\end{tabular}

Table 1: Table of adjoint representations. 
system of one-dimensional subalgebra for this case is given by $\left\{\Gamma_{2}, \Gamma_{3}, \Gamma_{2}\right.$ $\left.+b \Gamma_{3}\right\}$. In summary, the optimal system of one-dimensional subalgebras of the symmetry Lie algebra is

$$
\Theta=\left\{\Gamma_{1}, \Gamma_{2}, \Gamma_{3}, \Gamma_{1}+a \Gamma_{2}, \Gamma_{1}+b \Gamma_{3}, \Gamma_{2}+b \Gamma_{3}, \Gamma_{1}+a \Gamma_{2}+b \Gamma_{3}\right\} .
$$

Table 2 shows the solution of the invariant surface conditions (3.3) - (3.5) associated with the optimal system.

(i) $\operatorname{For}_{1}$, the characteristic

$$
\Phi=\left(\Phi_{\psi}, \Phi_{T}, \Phi_{C}\right),
$$

has the components

$$
\Phi_{\psi}=-\psi_{x}, \Phi_{T}=-T_{x}, \Phi_{C}=-C_{x} .
$$

Therefore, the general solutions of the invariant surface condition (3.3) - (3.5) are

$\psi=z_{1}(y), T=z_{2}(y), C=z_{3}(y)$.

Substitution from (3.32) into (2.15) yields

$u=\frac{d z_{1}}{d y}, v=0$.

This solution is not a particularly interesting one since it contradicts the boundary conditions. So, no solutions are invariant under the group generated by $\Gamma_{1}$.

(ii) For $\Gamma_{2}$, the characteristic (3.30) has the components

$$
\Phi_{\psi}=-\psi_{y}, \Phi_{T}=-T_{y}, \Phi_{C}=-C_{y}
$$

Therefore, the general solutions of the invariant surface condition (3.3) - (3.5) are

$$
\psi=j_{1}(x), T=j_{2}(x), C=j_{3}(x) .
$$

Substitution from (3.35) into (2.15) yields

$$
u=0, v=-\frac{d j_{1}}{d x} .
$$

This solution is not a particularly interesting one since it contradicts the boundary conditions. So, no solutions are invariant under the group generated by $\Gamma_{2}$.

(iii) For $\Gamma_{3}$, the characteristic (3.30) has the components

$\Phi_{\psi}=1, \Phi_{T}=0, \Phi_{C}=0$.

So, no solutions are invariant under the group generated by $\Gamma_{3}$.

(iv) For $\Gamma_{1}+a \Gamma_{2}$, the characteristic (3.30) has the components

$\Phi_{\psi}=-\psi_{x}-a \psi_{y}, \Phi_{T}=-T_{x}-a T_{y}, \Phi_{C}=-C_{x}-a C_{y}$.
Therefore, the general solutions of the invariant surface condition (3.3) - (3.5) are

$\psi=i_{1}(y-a x), T=i_{2}(y-a x), C=i_{3}(y-a x)$.

Substitution from (3.39) into (2.15) yields

$u=\frac{\partial i_{1}}{\partial y}, v=-b \frac{\partial i_{1}}{\partial x}$

Practically, Eq. (3.39) is a solution of the equations (2.9) - (2.12), even though it is not a particularly interesting one since it contradicts the boundary conditions. So, no solutions are invariant under the group generated by $\Gamma_{1}+a \Gamma_{2}$.

(v) For $\Gamma_{1}+b \Gamma_{3}$, the characteristic (50) has the components

$\Phi_{\psi}=-\psi_{y}, \Phi_{T}=-T_{x}, \Phi_{C}=-C_{x}$

Therefore, the general solutions of the invariant surface conditions (3.3)-(3.5) are

$\psi=b x+f_{1}(y), T=f_{2}(y), C=f_{3}(y)$.

Substitution from (3.42) into (2.15) yields

$u=\frac{d f_{1}}{d y}, v=-b$.

Substitution from (3.42) into (2.17) yields to two cases.

Case (1): $b^{2}-\frac{4 H e}{\operatorname{Pr}}>0$

$T=e^{m y}$,

where, $m=\frac{-b \operatorname{Pr}}{2}-\frac{\operatorname{Pr}}{2} \sqrt{b^{2}-\frac{4 H e}{\operatorname{Pr}}}$. Substitution from (3.44) into (2.18) and (2.16), respectively, yields

$\frac{d^{2} f_{3}}{d y^{2}}+S c\left[b-m \tau e^{m y}\right] \frac{d f_{3}}{d y}-m^{2} S c \tau e^{m y} f_{3}=0$

$\frac{d^{3} f_{1}}{d y^{3}}+b \frac{d^{2} f_{1}}{d y^{2}}+G c f_{3}+G r e^{m y}=0$.

Case (2): $b^{2}-\frac{4 H e}{\operatorname{Pr}}<0$

$T=e^{g_{y}} \cos (\gamma y)$,

where, $\vartheta=\frac{-b \operatorname{Pr}}{2}$ and $\gamma=\frac{\operatorname{Pr}}{2} \sqrt{\frac{4 H e}{\operatorname{Pr}}-b^{2}}$.

Substitution from (3.47) into (2.18) and (2.16), respectively, yields

\begin{tabular}{|l|l|l|}
\hline Generator & Characteristic $\Phi=\left(\Phi_{\psi}, \Phi_{T}, \Phi_{C}\right)$ & Solutions of the invariant surface conditions \\
\hline$\Gamma_{1}$ & $\Phi_{\psi}=-\psi_{x}, \Phi_{T}=-T_{x}, \Phi_{C}=-C_{x}$. & $\psi=z_{1}(y), T=z_{2}(y), C=z_{3}(y) \cdot$ \\
\hline$\Gamma_{2}$ & $\Phi_{\psi}=-\psi_{y}, \Phi_{T}=-T_{y}, \Phi_{C}=-C_{y}$. & $\psi=j_{1}(x), T=j_{2}(x), C=j_{3}(x)$. \\
\hline$\Gamma_{3}$ & $\Phi_{\psi}=1, \Phi_{T}=0, \Phi_{C}=0$. & No solution \\
\hline$\Gamma_{1}+a \Gamma_{2}$ & $\Phi_{\psi}=-\psi_{x}-a \psi_{y}, \Phi_{T}=-T_{x}-a T_{y}, \Phi_{C}=-C_{x}-a C_{y}$. & $\psi=i_{1}(y-a x), T=i_{2}(y-a x), C=i_{3}(a-b x)$. \\
\hline$\Gamma_{1}+b \Gamma_{3}$ & $\Phi_{\psi}=b-\psi_{x}, \Phi_{T}=-T_{x}, \Phi_{C}=-C_{x}$. & $\psi=b x+f_{1}(y), T=f_{2}(y), C=f_{3}(y)$. \\
\hline$\Gamma_{2}+b \Gamma_{3}$ & $\Phi_{\psi}=b-\psi_{y}, \Phi_{T}=-T_{y}, \Phi_{C}=-C_{y}$. & $\psi=b y+h_{1}(x), T=h_{2}(x), C=h_{3}(x)$. \\
\hline$\Gamma_{1}+a \Gamma_{2}+b \Gamma_{3}$ & $\Phi_{\psi}=b-\psi_{x}-a \psi y, \Phi_{T}=-T_{x}-a T_{y}, \Phi_{C}=-C_{x}-a C_{y}$. & $\psi=b x+L_{1}(y-a x), T=L_{2}(y-a x), C=L_{3}(y-a x)$. \\
\hline
\end{tabular}

Table 2: Solutions of the invariant surface conditions associated with the optimal system. 


$$
\left.\begin{array}{c}
\frac{d^{2} f_{3}}{d y^{2}}+S c\left[b-\tau e^{g_{y}}(\vartheta \cos (\gamma y)-\gamma \sin (\gamma y))\right] \frac{d f_{3}}{d y}- \\
\tau S c e^{g_{y}}\left[\left(\vartheta^{2}-\gamma^{2}\right) \cos (\gamma y)-2 \vartheta \gamma \sin (\gamma y)\right] f_{3}=0,
\end{array}\right\}
$$

(vi) For $\Gamma_{2}+b \Gamma_{3}$, the characteristic (3.30) has the components

$$
\Phi_{\psi}=b-\psi_{y}, \Phi_{T}=-T_{y}, \Phi_{C}=-C_{y} .
$$

Therefore, the general solutions of the invariant surface condition (3.3) - (3.5) are

$$
\psi=b y+h_{1}(x), T=h_{2}(x), C=h_{3}(x) .
$$

Substitution from (3.51) into (2.15) yields

$$
u=b, v=-\frac{d h_{1}}{d x} .
$$

This solution is not a particularly interesting one since it contradicts the boundary conditions. So, no solutions are invariant under the group generated by $\Gamma_{2}+b \Gamma_{3}$.

(vii) For $\Gamma_{1}+a \Gamma_{2}+b \Gamma_{3}$, the characteristic (3.30) has the components

$\Phi_{\psi}=b-\psi_{x}-a \psi_{y}, \Phi_{T}=-T_{x}-a T_{y}, \Phi_{C}=-C_{x}-a C_{y}$.

Therefore, the general solutions of the invariant surface condition (3.3) - (35) are

$$
\psi=b x+L_{1}(y-a x), T=L_{2}(y-a x), C=L_{3}(y-a x) .
$$

Substitution from (3.54) into (2.15) yields

$$
u=\frac{\partial L_{1}}{\partial y}, v=-b-\frac{\partial L_{1}}{\partial x} .
$$

Practically, Eq. (3.54) is a solution of the equations (2.9) - (2.12), even though it is not a particularly interesting one since it contradicts the boundary conditions. So, no solutions are invariant under the group generated by $\Gamma_{1}+a \Gamma_{2}+b \Gamma_{3}$.

\section{Results and Discussion}

\section{The effect of the heat generation parameter $\mathrm{He}$}

Figures 2 and 3 illustrate the horizontal velocity $\mathrm{u}$, temperature $\mathrm{T}$ and concentration $\mathrm{C}$ profiles for $\mathrm{G} c=1, \mathrm{G} r=1, \mathrm{P} r=1, \mathrm{~S} c=0.22$ and $\tau=1$ over a range of the heat generation parameter $\mathrm{He}$, for cases (1) and (2), respectively. For case (1), it is observed that the fluid temperature increases with the increase in the heat generation parameter He. Also the velocity boundary layer thickness increases with the increase in the thermal boundary layer, therefore the fluid velocity increases (Figure 2).

For case (2), the fluid temperature decreases with the increase in the heat generation parameter $\mathrm{He}$. The velocity boundary layer thickness decreases with the decrease in the thermal boundary layer which result in less induced flow along the plate therefore the fluid velocity decreases (Figure 3). A slight variation in the rate of increase of the concentration appears in both cases, as the heat generation parameter increases.

\section{The effect of Prandtl number Pr}

Figures 4 and 5 illustrate the horizontal velocity, temperature and concentration profiles for $\mathrm{G} c=1, \mathrm{G} r=1, \mathrm{~S} c=0.22$ and $\tau=1$ over a range of the Prandtl number $P_{r}$, case (1) for $H e=0.2$ and case (2) for $H e=1.5$, respectively. As seen, the temperature decreases as $\operatorname{Pr}$ increases.

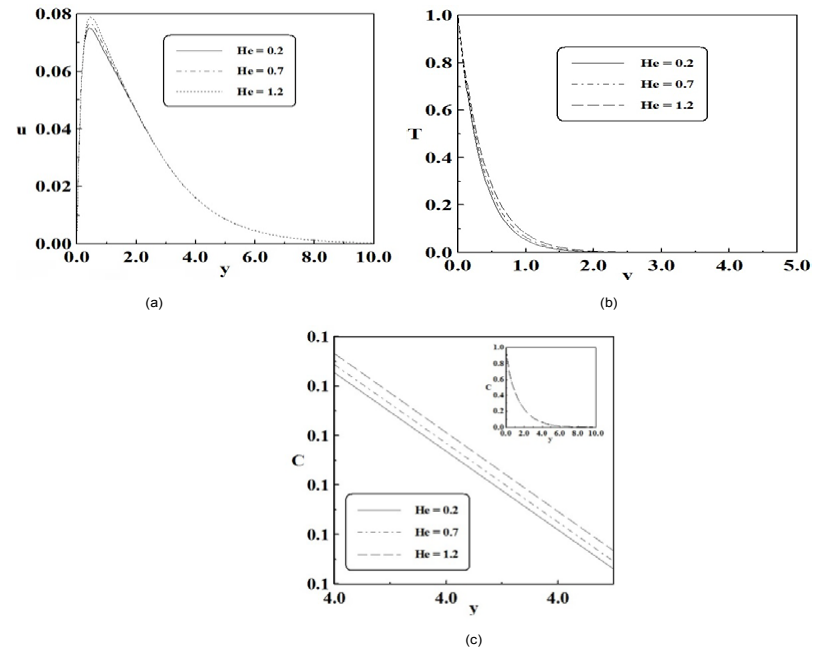

Figure 2: Effect of the heat generation parameter $\mathrm{He}$ on: (a) Horizontal velocity, (b) Temperature and (c) Concentration, for $G c=1, G r=1, P r=1$, Sc $=0.22$, and $\tau=1$ for case $(1)$.

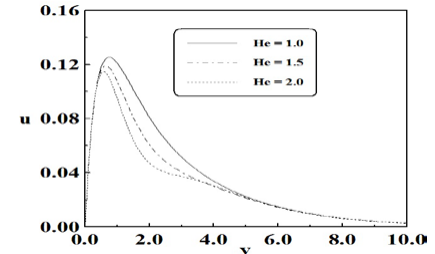

(a)

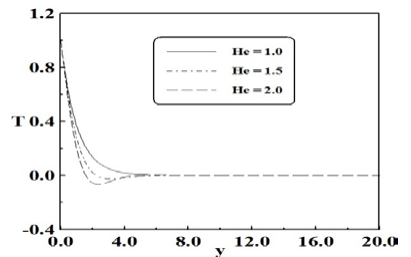

(b)

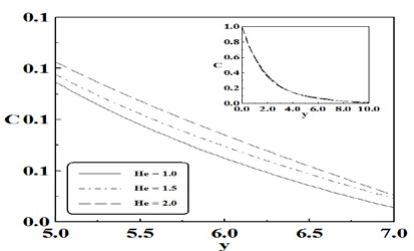

(c)

Figure 3: Effect of the heat generation parameter $\mathrm{He}$ on: (a) Horizontal velocity, (b) Temperature and (c) Concentration, for $G c=1, G r=1, \operatorname{Pr}=1$, Sc $=0.22, b=2$, and $\tau=1$ for case $(2)$.

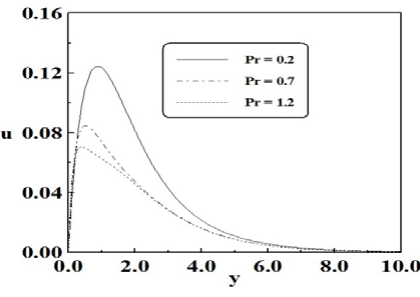

(a)

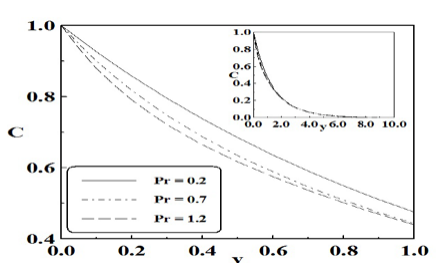

(c)

Figure 4: Effect of the Prandtl number $\operatorname{Pr}$ on: (a) Horizontal velocity, (b) Temperature and (c) Concentration, for $G c=1, \mathrm{G} r=1, \mathrm{Pr}=1, \mathrm{~S} c=0.22, b=3$ and $\tau=1$ for case $(1)$ at $H e=0.2$. 

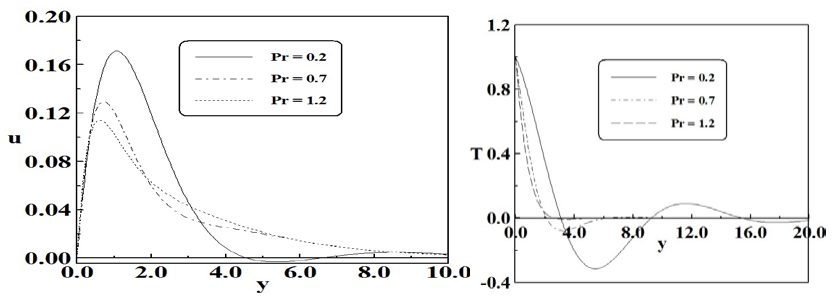

(a)

(b)

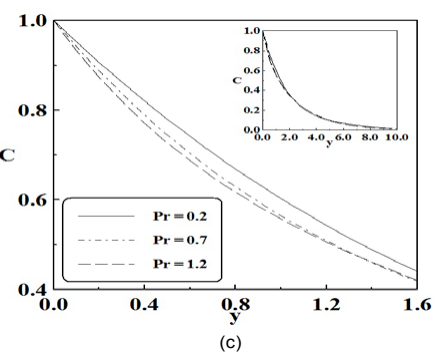

Figure 5: Effect of the Prandtl number Pron: (a) Horizontal velocity, (b) Temperature and (c) Concentration, for $\mathrm{G} c=1, \mathrm{G} r=1, \mathrm{Sc}=0.22, b=2$ and $\tau=1$ for case (2) at $H e=1.5$
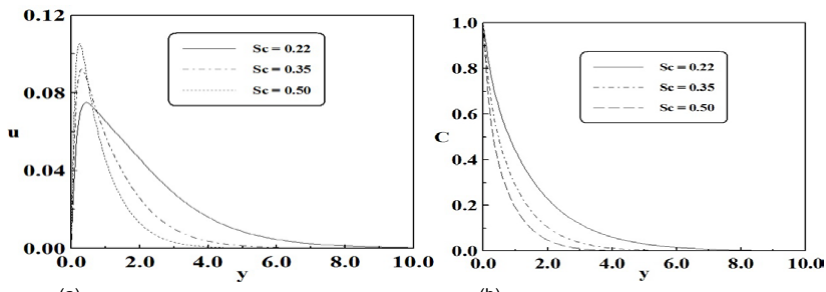

Figure 6: Effect of the Schmited number Scon: (a) Horizontal velocity and (b) Concentration, for $\mathrm{G} c=1, \mathrm{Gr}=1, \operatorname{Pr}=1, b=3$, and $\tau=1$ for case (1) at $\mathrm{He}=0.2$.

Physically $\operatorname{Pr}$ characterizes the ratio of thicknesses of the viscous and thermal boundary layers. The thermal boundary layer thickness decreases sharply with the increase in Pr which causes the fluid temperature to decrease. Also the velocity boundary layer thickness decreases with the decrease in the thermal boundary layer which result in less induced flow along the plate therefore the fluid velocity decrease. In both cases, a slight variation in the rate of decrease of the concentration appears as the Prandtl number Pr increases.

\section{The effect of Schimted number $S c$}

Figures 6 and 7 illustrate the horizontal velocity and the concentration profiles for $\mathrm{G} c=1, \mathrm{G} r=1, \mathrm{P} r=1$ and $\tau=1$ over a range of the Schmited number $S c$, case (1) for $H e=0.2$ and case (2) for $H e=1.5$, respectively. The concentration decreases as $S c$ increases. Physically $S c$ characterizes the ratio of thicknesses of the viscous and concentration boundary layers. The concentration boundary layer thickness decreases as the $S c$ increases therefore the fluid velocity decreases.

\section{The effect of thermo-phoretic parameter $\tau$}

Figures 8 and 9 illustrate the horizontal velocity and the concentration profiles for $\mathrm{G} c=1, \mathrm{G} r=1, \mathrm{P} r=1$ and $\mathrm{S} c=0.22$ over a range of the thermo-phoretic parameter $\tau$, case (1) for $H e=0.2$ and case (2) for $H e=1.5$, respectively. It is observed that the velocity and concentration of the fluid decrease with increase of $\tau$. In particular, the effect of increasing the parameter is limited to be increasing slightly the wall slope of the concentration profiles but decreasing the concentration.

\section{The effect of the solutal Grashof number $G c$}

Figure 10 illustrates the horizontal velocity profiles for $\mathrm{G} r=1, \mathrm{P} r=1$, $\mathrm{S} c=0.22$ and $\tau=1$, over a range of the solutal Grashof number $G c$, case (1) for $H e=0.2$ and case (2) for $H e=1.5$, respectively. As seen from both cases, the horizontal velocity increases as $G c$ increases. The Solutal Grashof number represents the relative importance of the buoyancy force to the viscous force. The increasing value of $G c$ implies the increasing strength of the flow which increase the velocity.

\section{The effect of thermal Grashof number $G r$}

Figure 11 illustrates the horizontal velocity profiles for $\mathrm{G} c=1, \mathrm{P} r=1$, $\mathrm{S} c=0.22$ and $\tau=1$, over a range of the thermal Grashof number $G r$, case (1) for $\mathrm{He}=0.2$ and case (2) for $H e=1.5$, respectively. We observe that, the velocity increases as $G r$ increases.

\section{Conculsion}

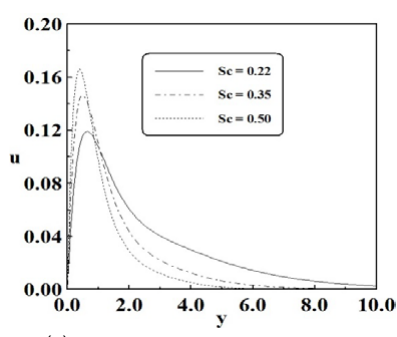

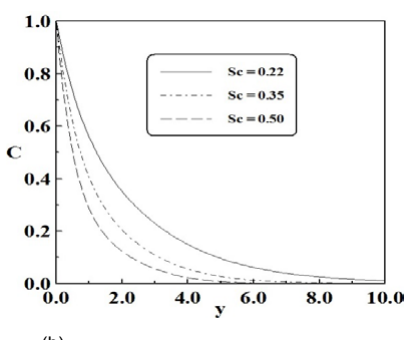

(b)
Figure 7: Effect of the Schmited number Scon: (a) Horizontal velocity and (b) Concentration, for $\mathrm{G} c=1, \mathrm{Gr}=1, \operatorname{Pr}=1, S c=0.22$ and $\tau=1$ for case (2) at $H e=1.5$.

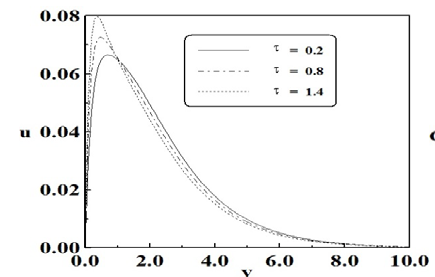

(a)

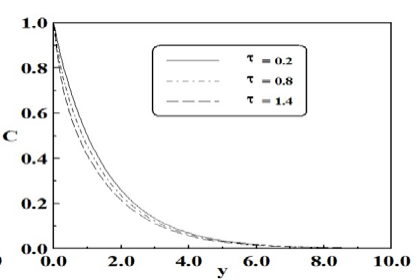

(b)
Figure 8: Effect of the thermo-phoretic parameter $\tau$ on: (a) Horizontal velocity and (b) Concentration, for $\mathrm{G} c=1, \mathrm{Gr}=1, \operatorname{Pr}=1, S_{c}=0.22$ and $b=3$ for case (1) at $H e=0.2$.

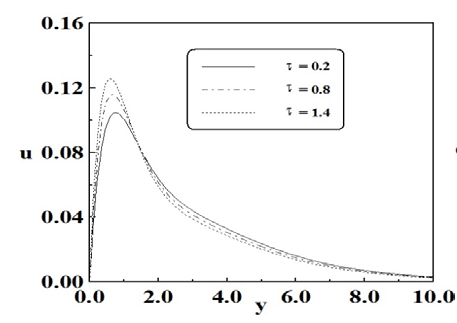

(a)

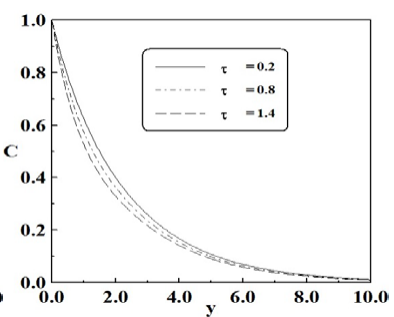

(b)
Figure 9: Effect of the thermo-phoretic parameter $\tau$ on: (a) Horizontal velocity and (b) Concentration, for $\mathrm{G} c=1, \mathrm{Gr}=1, \mathrm{Pr}=1, \mathrm{~S} c=0.22$ and $b=2$ for case (2) at $H e=1.5$. 


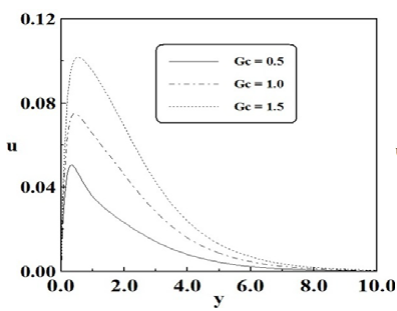

(a)

Figure 10: Effect of the solutal Grashof number Gc on the horizontal velocity, for $\mathrm{Gr}=1, \operatorname{Pr}=1, \mathrm{~S} c=0.22$, and $\tau=1$, (a) case (1) for $\mathrm{He}=0.2$ and $b=3$ (b) case (2) for $\mathrm{He}=1.5$ and $b=2$.

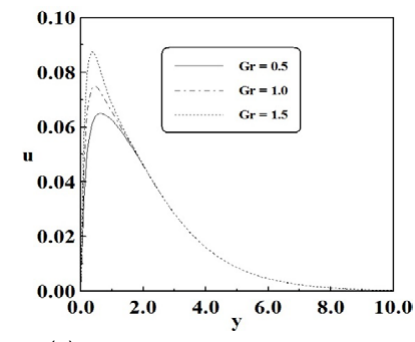

(a)

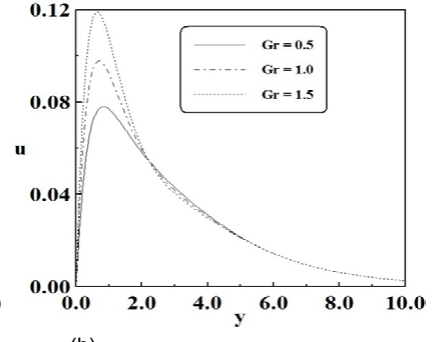

(b)
Figure 11: Effect of the thermal Grashof number Gr on the horizontal velocity, for $\mathrm{G} c=1, \mathrm{Pr}=1, \mathrm{~S} c=0.22$, and $\tau=1$, (a) case (1) for $H e=0.2$ and $b=3$ (b) case (2) for $\mathrm{He}=1.5$ and $b=2$.

The steady, laminar, two-dimensional free-convection boundary layer flow of a viscous incompressible fluid over a vertical plate with the effect of thermophoresis and heat generation has been investigated. The system of non-linear partial differential equations is solved using Liegroup method. The resulting ordinary differential equation from the energy equation is solved analytically and we have got exact solutions for the temperature of the fluid for two cases. The resulting ordinary differential equations obtained from the momentum and diffusion equations were solved numerically using using MATLAP package. The product of the manufacturing processes is affected by increasing or decreasing the physical parameters which appear in the governing equations that describe the physical phenomenon, which gives the significance of our results. It was found that the horizontal velocity decreased due to increases in the Prandtl number, Schimted number and thermo-phoretic parameter and it increased due to increases in the solutal Grashof number, thermal Grashof number. As increases in the heat generation parameter, the horizontal velocity increased in case (1) and it decreased in case (2). In addition, the temperature decreased due to increases in the Prandtl number. As increases in the heat generation parameter, the temperature increased in case (1) and it decreased in case (2). It was found that the particle concentration level as well as the concentration boundary layer thickness decreased due to increases Prandtl number, Schimted number and thermo-phoretic parameter. A slight variation in the rate of increase of the concentration appears as the heat generation parameter increases.

\section{Acknowledgment}

The authors are very thankful to the reviewers for their constructive and valuable comments.

\section{References}

1. Chamkha AJ, Al-Mudhaf AF, Pop I (2006) Effect of heat generation or absorption on thermophoretic free convection boundary layer from a vertical flat plate embedded in a porous medium. International Communications in Heat and Mass Transfer 33: 1096-1102.

2. Brock JR (1962) On the theory of thermal forces acting on aerosol particles. J Colloid Sci 17: 768-780.

3. Brock JR (1967) The thermal force in the transition region. J Colloid Interface Sci 23: 448-452.

4. Talbot L, Cheng RK, Schefer RW, Willis DR (1980) Thermophoresis of particles in a heated boundary layer. J Fluid Mech 101: 737-758.

5. Goren SL (1977) Thermophoresis of aerosol particles in the laminar boundary layer on a flat plate. J Colloid Interface Sci 61: 77-85.

6. Chiou MC (1998) Particle deposition from natural convection boundary layer flow onto an isothermal vertical cylinder. Acta Mechanica 129: 163-176.

7. Epstein M, Hauser GM, Henry RE (1985) Thermophoretic deposition of particles in natural convection flow from a vertical plate. Journal of Heat Transfer 107 272-276.

8. Jia G, Cipolla JW, Yener Y (1992) Thermophoresis of a radiating aerosol in laminar boundary layer flow. J Thermophys Heat Transfer 6: 476-482.

9. Chiou MC, Cleaver JW (1996) Effect of thermophoresis on sub-micron particle deposition from a laminar forced convection boundary layer flow onto an isothermal cylinder. J Aerosol Sci 27: 1155-1167.

10. Selim A, Hossain MA, Rees DAS (2003) The effect of surface mass transfer on mixed convection flow past a heated vertical flat permeable plate with thermophoresis. Int J Thermal Sci 42: 973-982.

11. Alam MS, Rahman MM, Sattar MA (2007) Similarity Solutions for Hydromagnetic Free Convective Heat and Mass Transfer Flow along a Semi-Infinite Permeable Inclined Flat Plate with Heat Generation and Thermophoresis. Nonlinear Analysis: Modelling and Control 12: 433-445.

12. Hill JM (1982) Solution of differential equations by means of one-parameter groups. Pitman Publishing INC, Massachusetts, USA.

13. Seshadri R, Na TY (1985) Group invariance in engineering boundary value problems. Springer-Verlag, New York.

14. Hydon PE (2000) Symmetry methods for differential equations. Cambridge University Press.

15. Burde GI (2002) Expanded Lie group transformations and similarity reductions of differential equations. Proceedings of Institute of Mathematics of NAS of Ukraine 43: 93-101.

16. Olver PJ (1986) Applications of Lie Groups to Differential Equations. New-York Springer-Verlag.

17. Boutros YZ, Abd-el-Malek MB, Badran NA, Hassan HS (2006) Lie-group method for unsteady flows in a semi-infinite expanding or contracting pipe with injection or suction through a porous wall. J Comput Appl Math 197: 465-494.

18. Boutros YZ, Abd-el-Malek MB, Badran NA, Hassan HS (2007) Lie-group method of solution for steady two-dimensional boundary-layer stagnation-point flow towards a heated stretching sheet placed in a porous medium. Meccanica 41: 681-691.

19. Boutros YZ, Abd-el-Malek MB, Badran NA, Hassan HS (2007) Lie-group method solution for two-dimensional viscous flow between slowly expanding or contracting walls with weak permeability. J Applied Mathematical Modelling 31: 1092-1108.

20. Abd-el-Malek MB, Badran NA, Hassan HS (2007) Lie-group method for predicting water content for immiscible flow of two fluids in a porous medium. Applied Mathematical Sciences 1: 1169-1180.

21. Abd-el-Malek MB, Hassan HS (2010) Symmetry analysis for steady boundarylayer stagnation-point flow of Rivlin-Ericksen fluid of second grade subject to suction. Nonlinear Analysis: Modelling and Control 15: 379-396.

22. Abd-el-Malek MB, Hassan HS (2010) Solution of Burgers' equation with time-dependent kinematic viscosity via Lie-group analysis. Proceedings of the 5th International Workshop "Group Analysis of Differential Equations and Integrable Systems", Protaras-Cyprus 6-14

23. Abd-el-Malek MB, Hassan HS (2014) Lie group method for solving the problem 
Citation: Abd-el-Malek MB, Badran NA, Hassan HS, Abbas HH (2015) Lie Group Method for Studying the Heat Generation Effect on Free-convection Laminar Boundary-layer Flow Over a Vertical Flat Plate. J Generalized Lie Theory Appl 9: 232. doi:10.4172/1736-4337.1000232

Page 9 of 9

of fission product behaviour in nuclear fuel. Math. Methods Appl Sci 37: 420-427.

24. Abd-el-Malek MB, Badran NA, Hassan HS, Abbas HH (2013) New solutions for solving the problem of particle trajectories in linear deep-water waves via Liegroup method. Appl Math Comput 219: 11365-11375.

\section{Jacobson N (1979) Lie algebras. New York, Dover}

26. Soh CW (2005) Invariant solutions of the unidirectional flow of an electrically charged power-law non-Newtonian fluid over a flat plate in presence of a transverse magnetic field. Commun Nonlinear Sci Numer Simul 10: 537-548. 